Supplementary Information for:

\title{
Multiplex digital microRNA detection using cross-inhibitory DNA circuits
}

\author{
Yannick Rondelez, Guillaume Gines*
}

Gulliver laboratory, ESPCI Paris - Université PSL. 10 rue Vauquelin, 75005 Paris
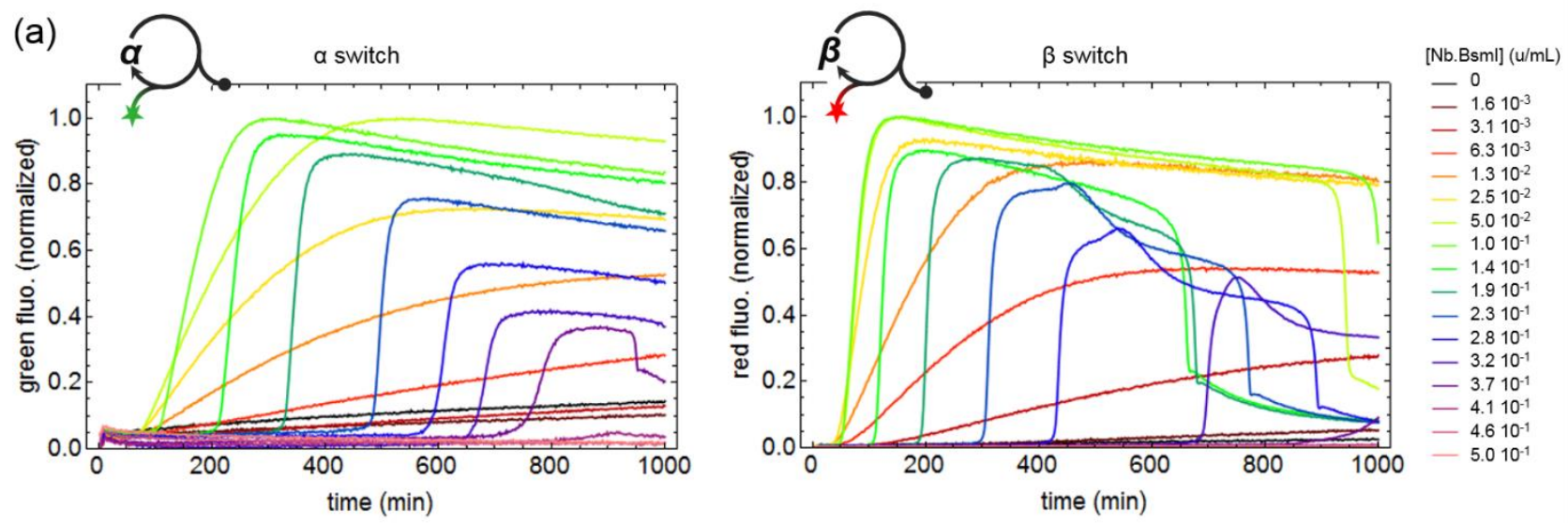

(b)

(c)
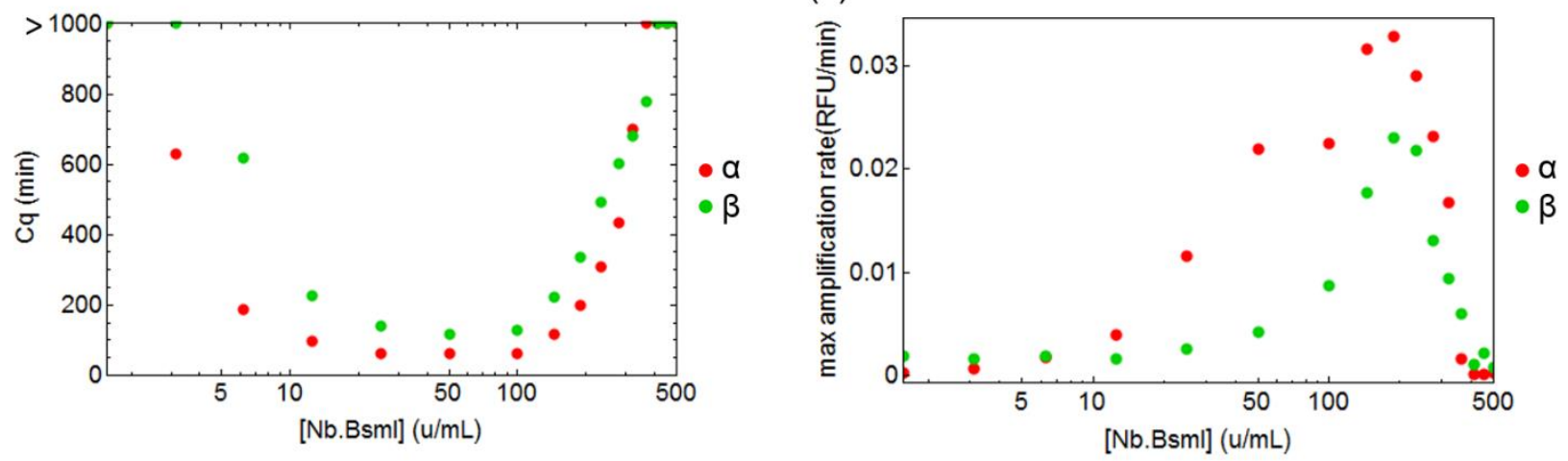

Supplementary Figure 1. Nb.BsmI effect on the self-start of $\alpha$ and $\beta$ switches. $50 \mathrm{nM}$ of aT and $5 \mathrm{nM}$ of the corresponding pT are incubated with an amplification mixture containing a varying concentration of nicking enzyme Nb.BsmI. (a) Amplification curves for the $\alpha$ and $\beta$ switches. (b) Cq are plotted as a function of $\mathrm{Nb}$.BsmI concentration. (c) Maximum amplification rates, extracted from the time traces derivatives are plotted as a function of $\mathrm{Nb}$.BsmI concentration. It can be noticed that at low concentration of $\mathrm{Nb} . \mathrm{BsmI}(<50 \mathrm{u} / \mathrm{mL})$, autocatalysis is not efficient and results in a transition from an exponential to a linear amplification process, as a direct consequence of the nicking rate becoming the limiting step. At higher concentration, the more the nicking enzyme, the later the amplification (higher $\mathrm{Cq}$ ). This effect might be attributed to a product inhibition effect: after nicking the duplex, the enzyme remains bound to its product, delaying the release of the output strands, therefore mitigating the accumulation of this trigger strand during the early phase of the amplification. From this experiment, it was decided to use $200 \mathrm{u} / \mathrm{mL}$ of Nb.BsmI in all experiments, which significantly delays nonspecific amplification, while allowing for a sharp exponential replication of $\alpha$ and $\beta$. The pseudotemplate concentration can be adjusted so that leak-triggered nonspecific amplification is not observed within 1000 minutes incubation. 
(a)

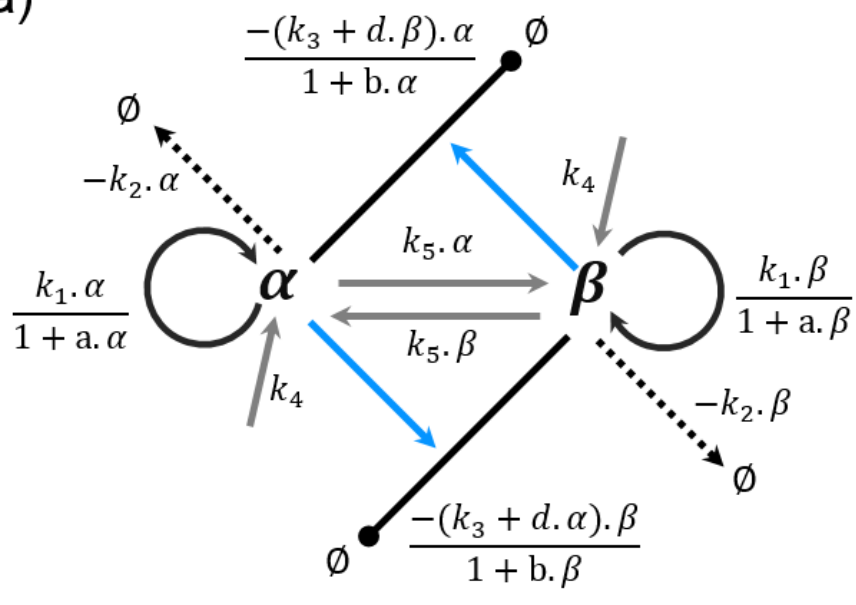

(b)

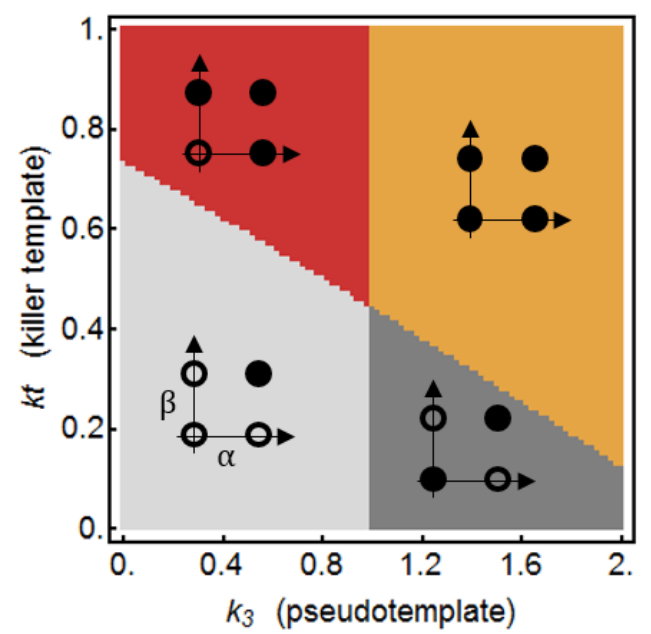

Supplementary Figure 2. Mathematical model of the duplex network. (a) Schematic of the simplified model. Each switch is represented by a single variable $\alpha$ or $\beta$. The associated kinetic terms are indicated next to each arrow. Template-controlled reaction are represented as black arrows (autocatalytic templates, pseudotemplates) and blue arrows (killer templates), and associated with Michaelis-Menten terms including a saturation constant. In particular, the saturable degradation pathway is represented with a disk-headed arrow. Its numerator combines a constant rate $\left(k_{3}\right.$, representing the constant pseudotemplate deactivation rate) and the killer template effect induced by the opposite switch (d. $\alpha$ or d. $\beta$ ). Leak reactions (spontaneous leak and crosstalk-induced leak) are shown as gray arrows and associated with zeroth or first order terms, respectively. The enzymatic degradation pathway is indicated with dashed arrows and associated with a first order term. For simplicity, the model is symmetric, with all constants associated to $\alpha$ equal to those associated to $\beta$. (b) The terms are combined in a two-variable ODE system and the model is numerically solved using the following values for the constants: $k_{1}=4, k_{2}=3, k_{4}=10^{-5}, k_{5}=10^{-2}, a=0.1, b=10$. The diagram shows the number of states that could be obtained starting from $\{\alpha[t=0], \beta[t=0]\} \in(\{0,0\},\{0,1\},\{1,0\},\{1,1\})$. Light gray: all trajectories converge to a single ON/ON state; Gray: two symmetrical states; Red, all ON states; Orange: all four states, representing the operational regime. The schematic phase space plots in inset show the attractive states as full disks, and unstable states as empty disks. Note that, in the orange area the higher the value of $k_{3}$, the less sensitive the switches will be. The model is provided as a Supplementary Mathematica file.

(a)
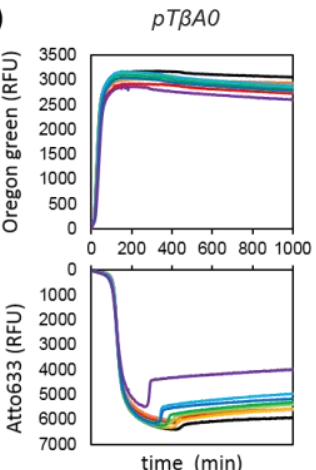

(b)

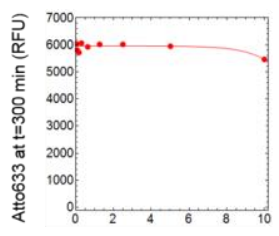

[kT] (nM)
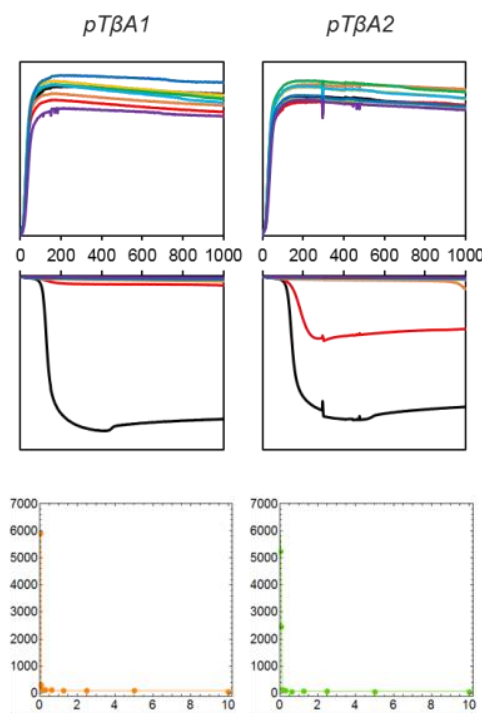

pTRA3
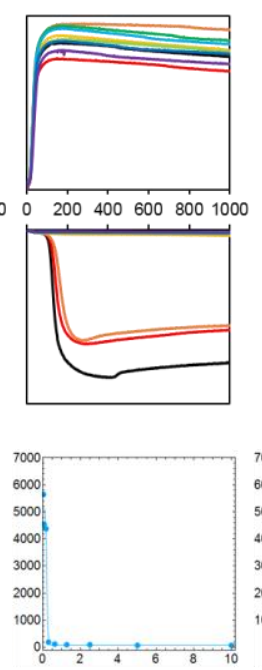

pTRA4
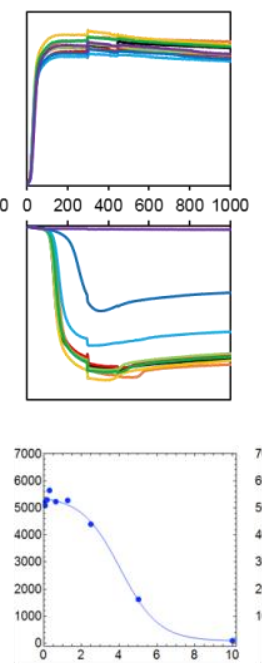

pT $\beta A 5$

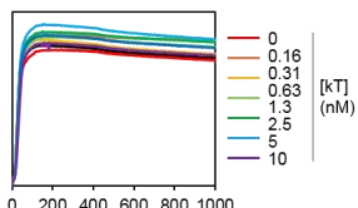

$200400600 \quad 8001000$
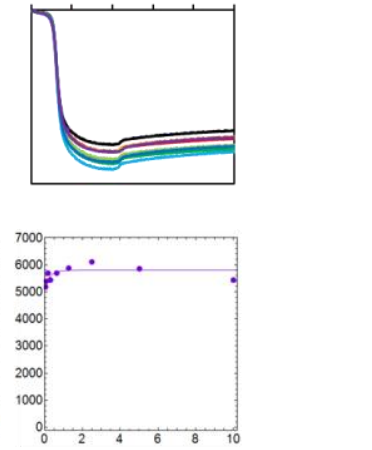

Supplementary Figure 3. Extended data from Figure 3. (a) Amplification curves for different concentrations of $\alpha \mathrm{k} \beta$ producing pT $\beta$ of different lengths. (b) Fluorescence of the beta switch $(t=300 \mathrm{~min})$ as a function of the $\alpha \mathrm{k} \beta$ concentration. 
(a)

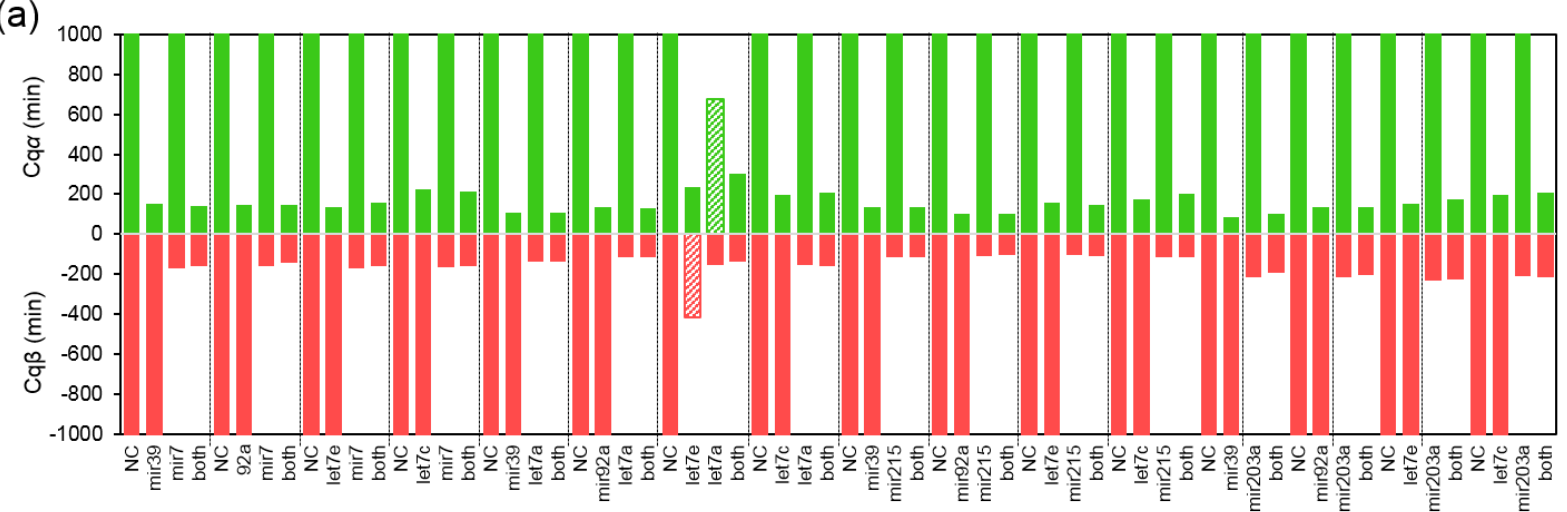

(b)

(c)

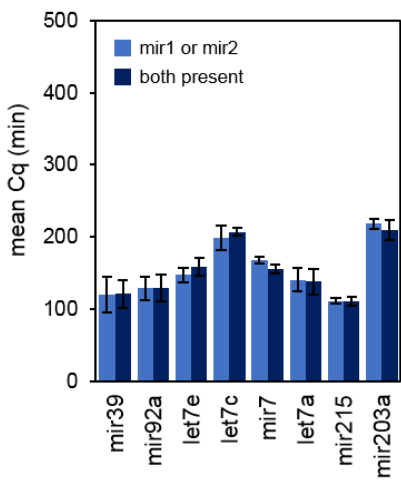
let7e
let7c
let7a
5' ugagguag 'agguuguauaguu
ugagguaguagguuguau ${ }^{g}$ guu $x$
ugagguaguagguuguauaguu
let7ato $\beta$
3' p ACTCCATCATCCAACATATCAAACTCAGTTTGCAGGTCTTACGT
let7e 5'ugagguag ${ }^{9}$ agguaguau ${ }^{a}$ guu $x$
let7c ugagguaguagguuguaugguu
let7a
let7ctoa
ugagguaguagguuguau ${ }^{\mathrm{a}} \mathrm{guu} \times$
3'p ACTCCATCATCCAACATACCAAACT CAGTTTGAAGTCCTAGGGT

\section{let7e \\ let7c \\ let7a}
let7etoa

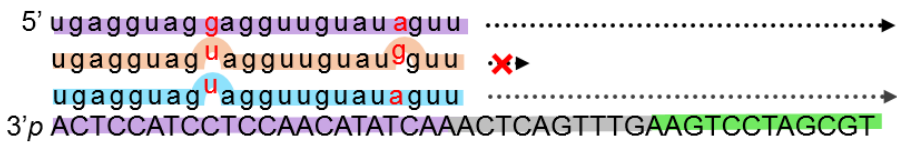

Supplementary Figure 4. Extended data from Figure 5c. (a) Amplification time (Cq) extracted for the 16 duplex experiments (0 or 10 pM of input target). Nonspecific amplification stemming from the high sequence homology in the let7e $(\alpha) / \operatorname{let} 7 \mathrm{a}(\beta)$ duplex assay is highlighted as hatched bars. (b) Mean Cq for the 8 different targets, either spiked alone ("mir1 or mir2") or with the second target ("both present"). (c) Sequence complementary between let7e, let7c or let7a and their converter templates. This simplified sketch suggests that let7e can be extended by the polymerase on the let7a cT and conversely, resulting in the observed lack of selectivity for these sequences. On the contrary, $3^{\prime}$ mismatching targets like let $7 \mathrm{c} \mathrm{do} \mathrm{not} \mathrm{interfere}$ with let7a (or let7e) cT, as the polymerase may not be able to extend from unmatched 3 ' sequences.

(a)

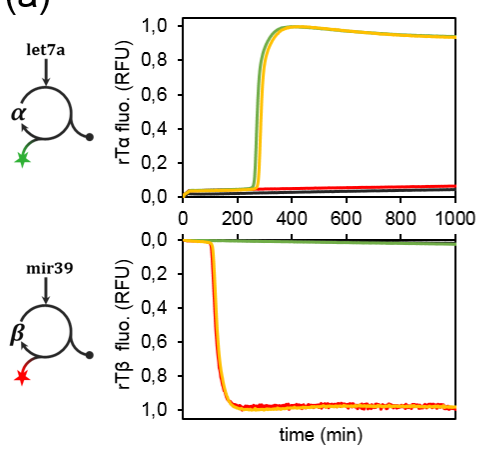

(b)

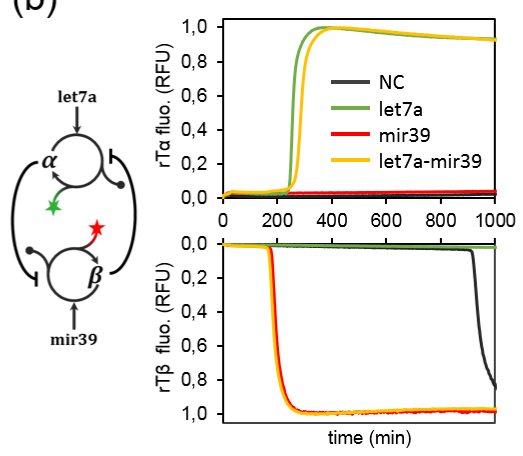

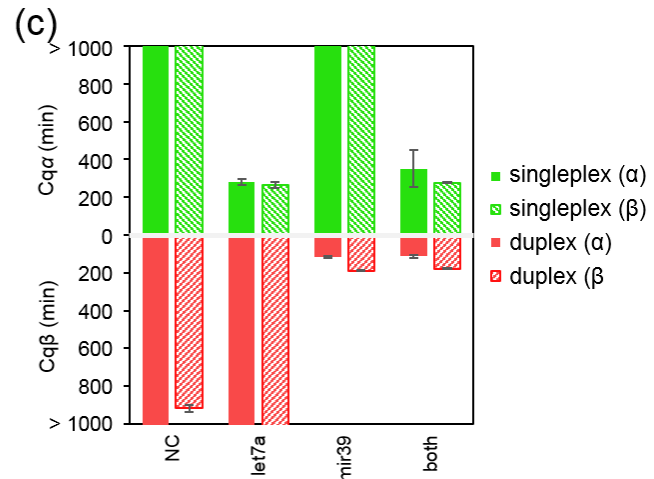

Supplementary Figure 5. In-solution singleplex versus duplex assay. (a) Amplification curves of $\alpha$ (top) and $\beta$ (bottom) bistable circuits incubated separately with the amplification mix and 0 or 3 pM of let7a and mir39 targets (singleplex assay). (b) Amplification curve of $\alpha$ and $\beta$ switches embedded in the full tetrastable circuit (duplex assay). (c) Amplification time ( $\mathrm{Cq}$ ) measured for a duplicate experiment. From these data, we concluded that the killer templates have little effect on the amplification time in these conditions. 
(a)
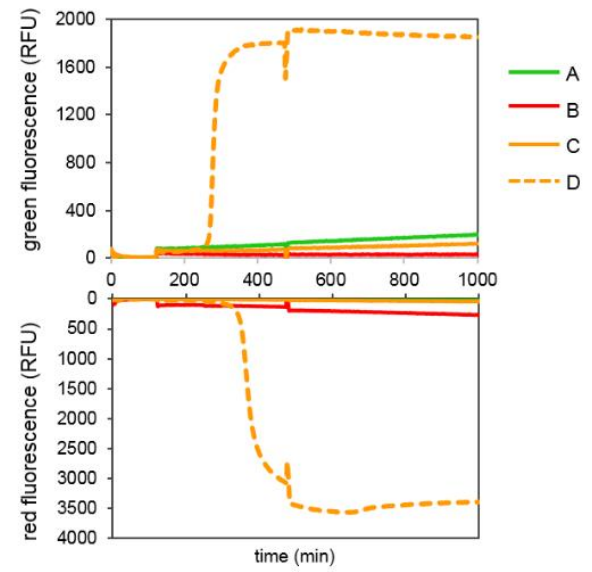

(b)

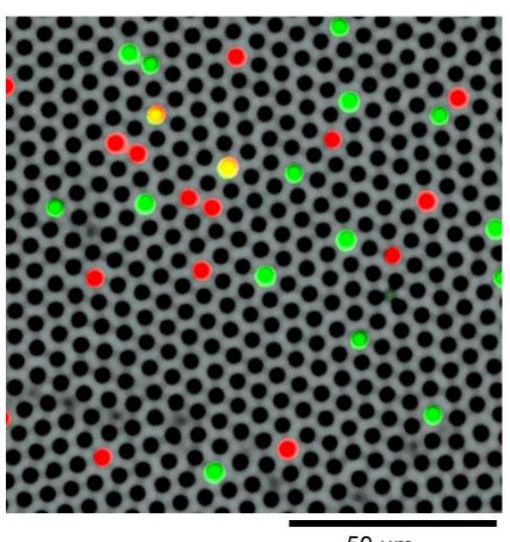

$50 \mu \mathrm{m}$

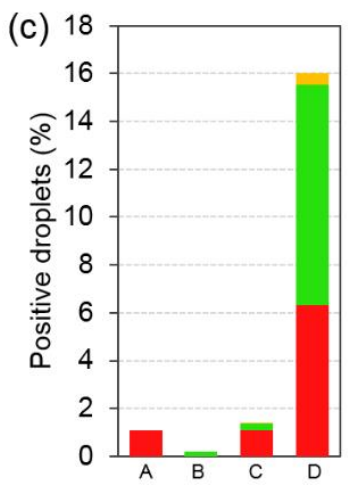

m red $=$ green $m$ orange

(d)
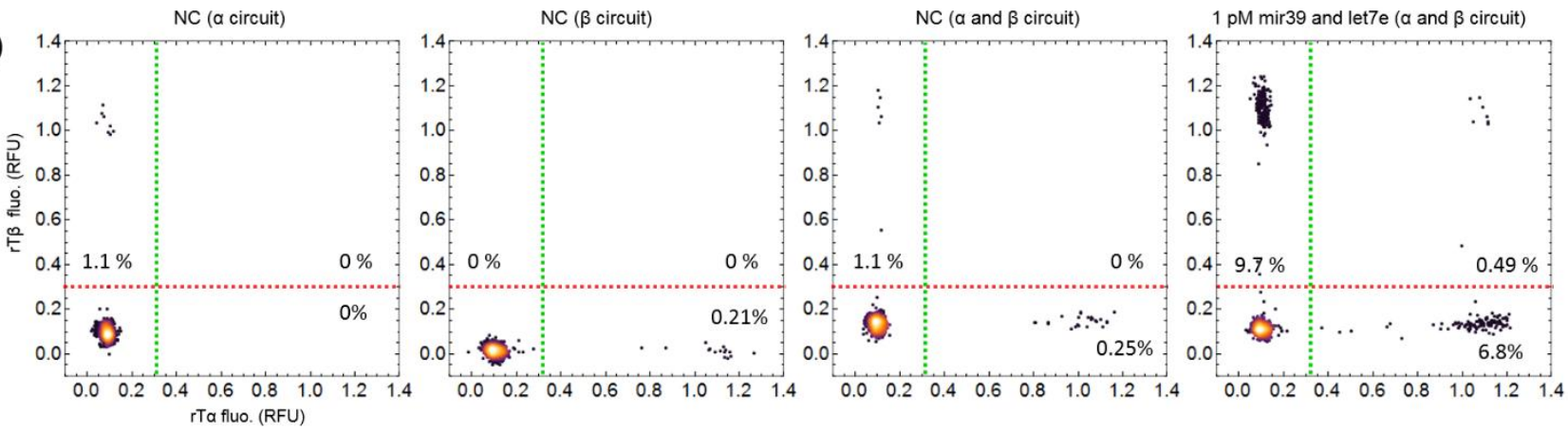

Supplementary Figure 6. Limit of blank for singleplex versus duplex assay. 4 samples are assembled as follow: sample $A=\alpha$ circuit only, 0 pM target, sample $B=\beta$ circuit only, 0 pM target, sample $C=\alpha$ and $\beta$ circuit, 0 pM target, sample $D=\alpha$ and $\beta$ circuit, 1 pM target mir39 and let7e. (a) Amplification curves of the 4 samples in solution. (b) Composite image of a portion of the microfluidics chamber (brightfield, green and red fluorescence). (c) $2 \mathrm{D}$ histograms of the droplets fluorescence $(\alpha$ switch $=$ green fluorescence, $\beta$ switch $=$ red fluorescence). (d) Percentage of positive droplets. The percentage of false positive droplets is qualitatively similar whether the assay is performed in singleplex or duplex (false positive $\alpha=1.1 \%$ singleplex and $1.1 \%$ in duplex, false positive $\alpha=0.25 \%$ singleplex and $0.28 \%$ in duplex). 
(a)
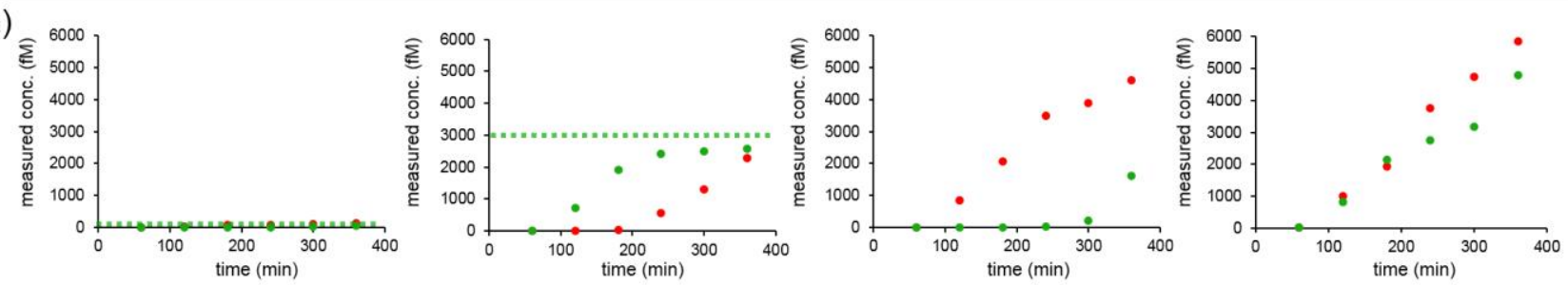

(b)
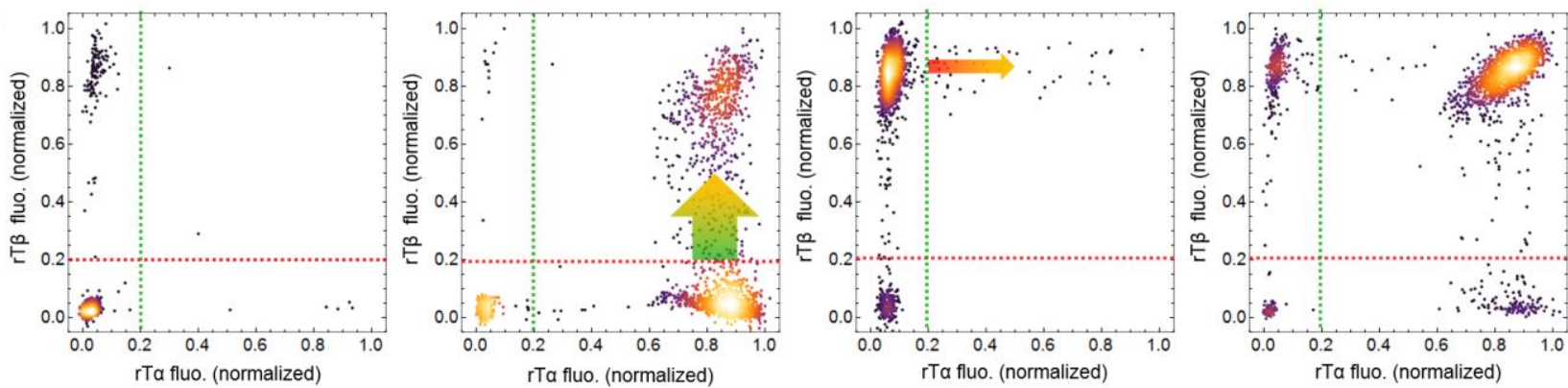

Supplementary Figure 7. Digital duplex assay $(\mathrm{mir} 39(\alpha) /$ let7a $(\beta)$ in the absence of killer templates (from left to right, expected concentrations of mir39 and let7a (fM) are 0:0, 3000:0, 0:3000 and 3000:3000). (a) Measured concentrations as a function of time. Green and red lines correspond to the expected concentrations of mir39 and let7a respectively. (b) $2 \mathrm{D}$ histograms of the droplets' fluorescence after 240 minutes of incubation. As expected, crosstalk reactions between the switches result in a substantial fraction of false double positive droplets (top right quadrant), migrating from the monocolor populations. This effect is markedly more important for the alpha switch, which self-triggers soon after the amplification of the beta switch, and hampering the accurate quantification of let7a in this duplex assay.

(a)

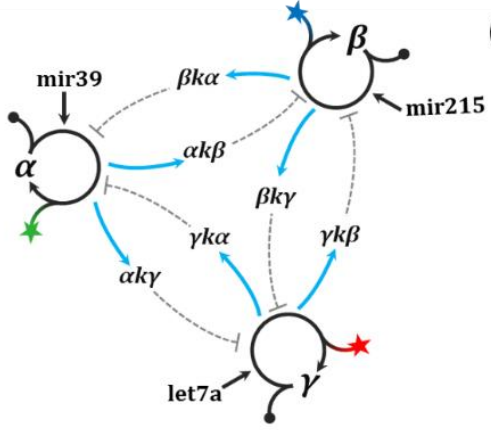

(c)

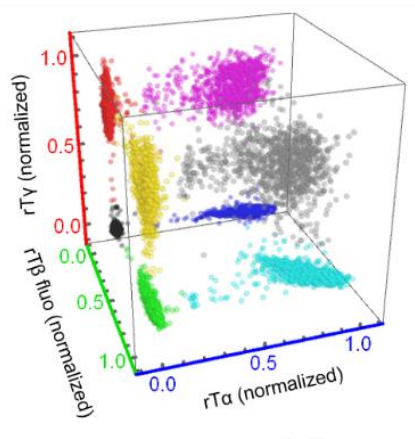

(b)

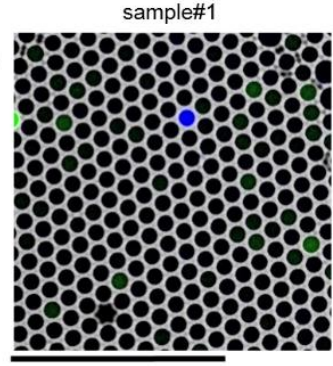

sample\#2

sample\#3

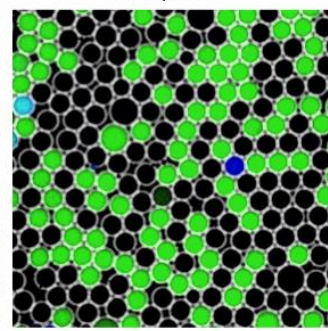

(d)
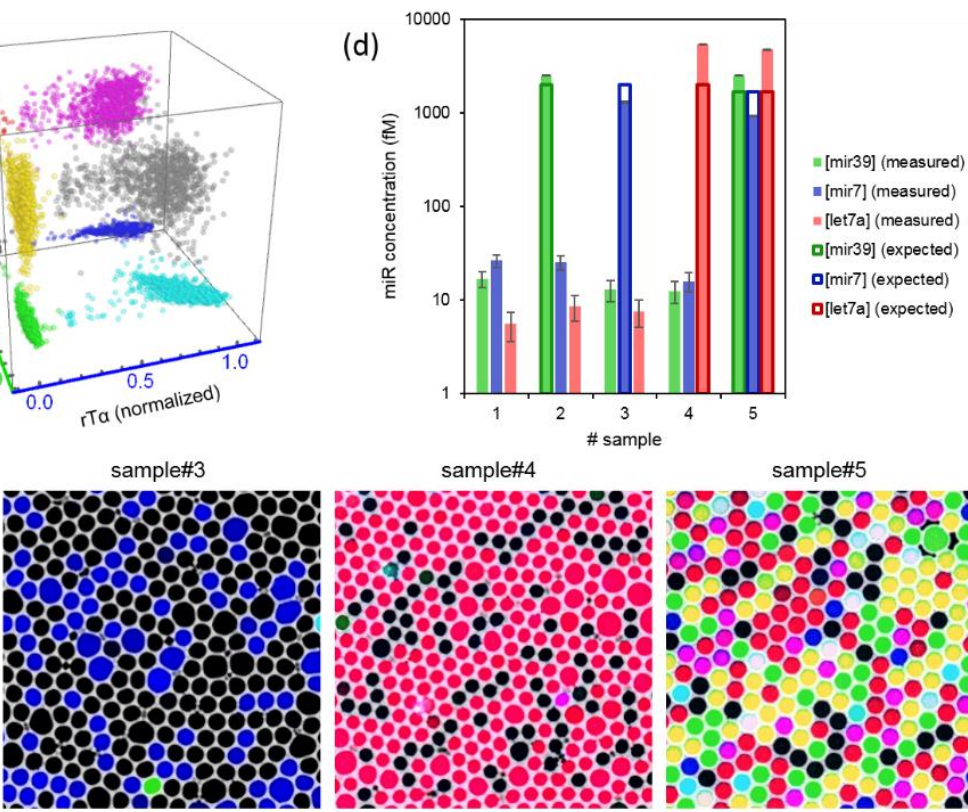

sample\#4

sample\#5

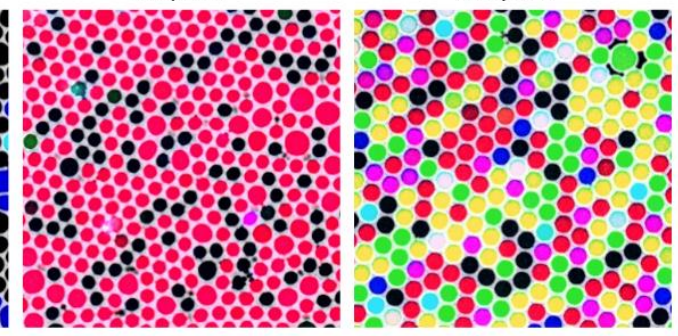

Supplementary Figure 8. Digital triplex assay $(\operatorname{mir} 39(\alpha) / \operatorname{mir} 7(\beta) /$ let7a $(\gamma))$. (a) The triplex octastable DNA circuit comprises 18 templates $(30 \mathrm{nM}$ aT $\alpha, 30 \mathrm{nM} \mathrm{aT} \beta, 30 \mathrm{nM}$ aT $\gamma, 6.5 \mathrm{nM} \mathrm{pT} \alpha, 6.5 \mathrm{nM} \mathrm{pT} \beta, 4 \mathrm{nM} \mathrm{pT} \gamma, 30 \mathrm{nM} \mathrm{rT} \alpha$ (Oregon green dye), $30 \mathrm{nM}$ aT $\beta$ (Сy3.5 dye), $30 \mathrm{nM}$ aT $\gamma$ (Cy5 dye), $0.5 \mathrm{nM}$ mir39to $\alpha, 0.5 \mathrm{nM}$ mir7to $\beta, 0.5 \mathrm{nM}$ let7ato $\gamma, 1.2 \mathrm{nM} \alpha \mathrm{k} \beta, 3 \mathrm{nM} \beta \mathrm{k} \alpha, 1.5 \mathrm{nM} \alpha \mathrm{k} \gamma, 1 \mathrm{nM} \gamma \mathrm{k} \alpha, 0.3 \mathrm{nM} \beta \mathrm{k} \gamma, 1.2 \mathrm{nM} \gamma \mathrm{k} \beta$ ). 5 samples were prepared with the following concentrations of mir39:mir215:let7a (in fM): sample\#1 = 0:0:0, sample\#2 = 2000:0:0 , sample\#3 = 0:2000:0, sample\#4 = 0:0:2000, sample\#5 = 1660:1660:1660. (b) Composite images (green = OregonGreen488, Blue $=$ Cy3.5, Red $=$ Cy5) of microscopy snapshots for the 5 samples following amplification (scale bar $=100 \mu \mathrm{m}$ ). (c) 3D plot of the droplets fluorescence in the three reporting channels, for sample\#5. It shows $2^{3}=8$ clusters, as expected. (d) Measured versus expected concentrations for the three targeted microRNAs. 

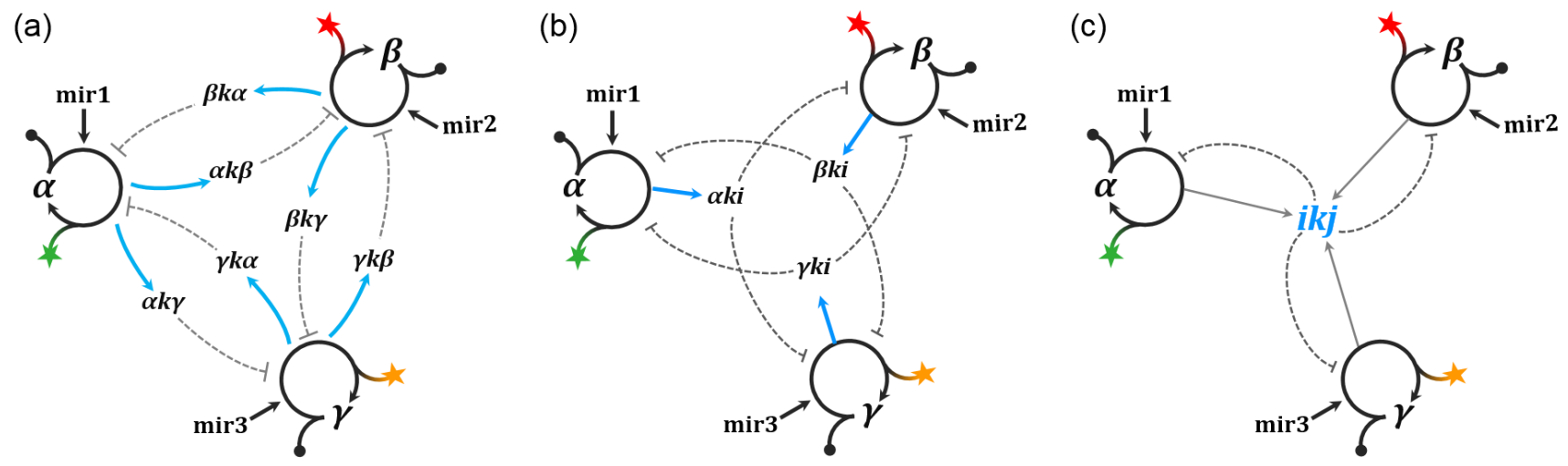

Supplementary Figure 9. Scaling-up strategies for multiplex digital assays. (a) The strategy of the present study consists in cross-inhibitory circuits, where each node is connected to all others and reciprocally using killer templates (e.g. $\alpha k \beta$ ). Each blue arrow represents a killer template and dashed lines show the inhibitors' target(s). Here, $n$. ( $n-1) \mathrm{kT}$ are required to connect all nodes (where $n$ is the number of targets). (b) It may be possible to cut the number of $\mathrm{kT}$ down to $n$ by designing pseudo-universal inhibitor. In this case, each kT (e.g. $\alpha k i)$ accepts as input the corresponding trigger ( $\alpha$, $\beta$ or $\gamma$ in this 3-node system), and in turn, outputs an inhibitor strand that represses all other switches. This may be implemented using partially degenerated output sequence that would target all nodes simultaneously. (c) Ideally, one may think about a universal inhibition mechanism where target-triggered switched repress all others via a common, single pathway $i \mathrm{kj}$. A possible strategy would be to provide the system with a limited amount of substrate such as dNTPs. Once triggered, the autocatalytic reaction would quickly consume the dNTP, causing a depletion of resources that would prevent all untriggered switches to start. 
Supplementary Table 1. Extended data from Figure 6. Raw data obtained from the image analysis of the 30 samples.

\begin{tabular}{|c|c|c|c|c|c|c|c|c|c|c|c|c|c|}
\hline Sample & $\begin{array}{l}\text { expected conc. } \\
\text { mir1 ( } \mathrm{fM})\end{array}$ & $\begin{array}{l}\text { expected conc. } \\
\text { mir2 (fM) }\end{array}$ & $\begin{array}{l}\text { volume } \\
(\mathrm{pL})\end{array}$ & $\begin{array}{l}\text { droplets } \\
\text { analyze }\end{array}$ & \begin{tabular}{|l|} 
\#green \\
droplets
\end{tabular} & $\begin{array}{l}\text { \#red } \\
\text { droplets }\end{array}$ & $\begin{array}{l}\text { \# orange } \\
\text { droplets }\end{array}$ & $\begin{array}{l}\text { \% green } \\
\text { droplets }\end{array}$ & $\begin{array}{l}\text { \% red } \\
\text { droplets }\end{array}$ & $\begin{array}{c}\% \text { orange } \\
\text { droplets }\end{array}$ & $\begin{array}{l}\text { measured conc. } \\
\text { mir1 (fM) }\end{array}$ & \multicolumn{2}{|c|}{$\begin{array}{l}\text { measured conc. mir2 } \\
\text { (fM) }\end{array}$} \\
\hline 1 & mir92a 0 & let7a 500 & 0.32 & 14948 & 0 & 738 & 0 & $0.0 \%$ & $4.9 \%$ & $0.0 \%$ & $0 \pm 0$ & $261 \pm$ & 18 \\
\hline 2 & mir92a 2500 & let7a 0.5 & 0.32 & 20701 & 5846 & 1134 & 131 & $28.2 \%$ & $5.5 \%$ & $1.5 \%$ & $1711 \pm 32$ & $291 \pm$ & 16 \\
\hline 3 & mir92a 0 & mir7 300 & 0.32 & 22559 & 2 & 1076 & 0 & $0.0 \%$ & $4.8 \%$ & $0.0 \%$ & $0 \pm 1$ & $252 \pm$ & 14 \\
\hline 4 & mir92a 1000 & mir7 1000 & 0.52 & 5231 & 2145 & 1030 & 317 & $41.0 \%$ & $19.7 \%$ & $8.1 \%$ & $1671 \pm 42$ & $695 \pm$ & 34 \\
\hline 5 & mir92a 0 & $\operatorname{mir} 70$ & 0.27 & 15135 & 138 & 181 & 10 & $0.9 \%$ & $1.2 \%$ & $0.0 \%$ & $57 \pm 9$ & $74 \pm$ & 11 \\
\hline 6 & mir92a 0 & mir7 1000 & 0.27 & 11022 & 95 & 1233 & 8 & $0.9 \%$ & $11.2 \%$ & $0.1 \%$ & $54 \pm 11$ & $734 \pm$ & 37 \\
\hline 7 & mir92a 1000 & mir7 0 & 0.27 & 11479 & 1547 & 224 & 29 & $13.5 \%$ & $2.0 \%$ & $0.3 \%$ & $895 \pm 39$ & $122 \pm$ & 16 \\
\hline 8 & mir92a 1000 & mir7 1000 & 0.27 & 19556 & 2565 & 2107 & 242 & $13.1 \%$ & $10.8 \%$ & $1.4 \%$ & $870 \pm 29$ & $705 \pm$ & 27 \\
\hline 9 & mir92a 1000 & mir7 5000 & 0.24 & 18800 & 2317 & 8790 & 970 & $12.3 \%$ & $46.8 \%$ & $5.8 \%$ & $912 \pm 33$ & $4373 \pm$ & 50 \\
\hline 10 & mir92a 5000 & mir7 1000 & 0.22 & 20443 & 7198 & 2387 & 758 & $35.2 \%$ & $11.7 \%$ & $4.1 \%$ & $3258 \pm 49$ & $932 \pm$ & 33 \\
\hline 11 & mir92a 1000 & mir7 1000 & 0.18 & 34849 & 3382 & 2360 & 140 & $9.7 \%$ & $6.8 \%$ & $0.7 \%$ & $943 \pm 29$ & $648 \pm$ & 24 \\
\hline 12 & Let7e 2000 & mir39 0 & 1.29 & 7627 & 6017 & 118 & 92 & $78.9 \%$ & $1.5 \%$ & $1.2 \%$ & $2002 \pm 12$ & $20 \pm$ & 4 \\
\hline 13 & Let7e 0 & mir39 2000 & 1.29 & 8656 & 454 & 7905 & 418 & $5.2 \%$ & $91.3 \%$ & $4.8 \%$ & $69 \pm 6$ & $3147 \pm$ & 8 \\
\hline 14 & Let7e 2000 & mir39 2000 & 1.29 & 12048 & 8726 & 9346 & 6880 & $72.4 \%$ & $77.6 \%$ & $56.2 \%$ & $1658 \pm 10$ & $1924 \pm$ & 10 \\
\hline 15 & mir39 0 & Let7a 0 & 0.52 & 29897 & 153 & 346 & 3 & $0.5 \%$ & $1.2 \%$ & $0.0 \%$ & $16 \pm 3$ & $37 \pm$ & 4 \\
\hline 16 & mir39 0 & Let7a 3000 & 0.52 & 14427 & 67 & 7941 & 29 & $0.5 \%$ & $55.0 \%$ & $0.3 \%$ & $15 \pm 4$ & $2532 \pm$ & 26 \\
\hline 17 & mir39 3000 & Let7a 0 & 0.52 & 8818 & 4679 & 104 & 33 & $53.1 \%$ & $1.2 \%$ & $0.6 \%$ & $2396 \pm 33$ & $38 \pm$ & 7 \\
\hline 18 & mir39 0 & Let7a 0 & 0.52 & 6565 & 21 & 112 & 3 & $0.3 \%$ & $1.7 \%$ & $0.0 \%$ & $10 \pm 4$ & $55 \pm$ & 10 \\
\hline 19 & mir39 0 & Let7a 3000 & 0.52 & 3409 & 9 & 2178 & 1 & $0.3 \%$ & $63.9 \%$ & $0.2 \%$ & $8 \pm 5$ & $3226 \pm$ & 51 \\
\hline 20 & mir39 0 & Let7a 0 & 1.29 & 6202 & 3 & 38 & 1 & $0.0 \%$ & $0.6 \%$ & $0.0 \%$ & $1 \pm 1$ & $8 \pm$ & 3 \\
\hline 21 & mir39 0 & Let7a 3000 & 1.29 & 2148 & 5 & 1875 & 4 & $0.2 \%$ & $87.3 \%$ & $0.2 \%$ & $3 \pm 3$ & $2655 \pm$ & 18 \\
\hline 22 & mir39 3000 & Let7a 0 & 1.29 & 4449 & 4098 & 125 & 117 & $92.1 \%$ & $2.8 \%$ & $2.6 \%$ & $3269 \pm 10$ & $37 \pm$ & 6 \\
\hline 23 & mir39 3000 & Let7a 3000 & 1.29 & 19802 & 18209 & 16684 & 15380 & $92.0 \%$ & $84.3 \%$ & $77.5 \%$ & $3244 \pm 5$ & $2380 \pm$ & 7 \\
\hline 24 & mir39 0 & let7a 2000 & 0.34 & 22041 & 177 & 9720 & 45 & $0.8 \%$ & $44.1 \%$ & $0.4 \%$ & $39 \pm 6$ & $2797 \pm$ & 32 \\
\hline 25 & mir39 2000 & let7a 0 & 0.34 & 15684 & 4603 & 256 & 42 & $29.3 \%$ & $1.6 \%$ & $0.5 \%$ & $1671 \pm 34$ & $79 \pm$ & 10 \\
\hline 26 & mir39 1000 & let7a 1000 & 0.34 & 17768 & $3.0 \mathrm{E}+03$ & 4222 & 3.7E+02 & $17.1 \%$ & $23.8 \%$ & $4.1 \%$ & $901 \pm 27$ & $1305 \pm$ & 30 \\
\hline 27 & let7e 0 & mir215 0 & 0.43 & 5937 & 28 & 65 & 3 & $0.5 \%$ & $1.1 \%$ & $0.0 \%$ & $18 \pm 7$ & $42 \pm$ & 10 \\
\hline 28 & let7e 500 & mir215 1000 & 0.43 & 21322 & 4101 & 6032 & 890 & $19.2 \%$ & $28.3 \%$ & $5.4 \%$ & $815 \pm 20$ & $1268 \pm$ & 23 \\
\hline 29 & let7e 1000 & mir215 500 & 0.43 & 18071 & 6363 & 3134 & 793 & $35.2 \%$ & $17.3 \%$ & $6.1 \%$ & $1655 \pm 27$ & $726 \pm$ & 21 \\
\hline 30 & let7e 100 & mir215 500 & 0.43 & 13208 & 979 & 1532 & 75 & $7.4 \%$ & $11.6 \%$ & $0.9 \%$ & $294 \pm 17$ & $470 \pm$ & 21 \\
\hline
\end{tabular}


Supplementary Table 2. Oligonucleotide sequences used throughout this study $(\mathrm{a} T=$ amplification template, $\mathrm{pT}=\mathrm{pseudotemplate}, \mathrm{rT}=$ reporting template, $\mathrm{cT}=$ conversion template, $\mathrm{kT}=$ killer template). “*” denotes phosphorothioate backbone modification as a protection against exonuclease depolymerization. " $p$ " denotes 3' phosphate modification to prevent from unspecific template extension. "OregonGree488”, "Atto633", "BMNQ535" and "BHQ2" denote 5' fluorophores and 3' quenchers respectively. Nucleotides in bold font and dashes represent nicking recognition sites and cutting sites (Nt.BstNBI and Nb.BsmI) on the complementary strand respectively. Upper and lower cases represent deoxyribonucleotides and ribonucleotides respectively. The color code aims at highlighting the $\alpha$ (green), $\beta$ (red), $\gamma$ (blue) and pseudotemplate extension (pink) sequences (see Figure 2).

\begin{tabular}{|c|c|c|}
\hline ID & Sequence & Function \\
\hline$\alpha$ & CATTCAGGATCG & trigger \\
\hline$\beta$ & CATTCTGGACTG & trigger \\
\hline Y & CATTCATCCCAG & trigger \\
\hline $\mathrm{aT \alpha}$ & C*G*A*TCCTGAATG-CGATCCTGAA $p$ & aT \\
\hline aT $\beta$ & C*A*G*TCCAGAATG-CAGTCCAGAA $p$ & $\mathrm{aT}$ \\
\hline aTy & C*T*G*GGATGAATG-CTGGGATGAA $p$ & aT \\
\hline $\mathrm{pT} \alpha$ & T*T*T*TTCGATCCTGAATG $p$ & pT \\
\hline рT $\beta$ & T*T*T*TTCAGTCCAGAATG $p$ & pT \\
\hline pTy & T*T*T*TT CTGGGATGAATG $p$ & pT \\
\hline $\mathrm{rT \alpha}$ & OregonGreen488 *A*T*TCAGAATG-CGATCCTGAAT BMNQ535 & $\mathrm{rT}$ \\
\hline $\mathrm{rT \alpha}-\mathrm{Cy} 3.5$ & Cy3.5 *A*T*TCAGAATG-CGATCCTGAAT BHQ2 & $\mathrm{rT}$ \\
\hline rT $\beta$ & Atto633 *A*T*TCTGAATG-CAGTCCAGAAT BHQ2 & $\mathrm{rT}$ \\
\hline rTy & Cy5 *C*T*TCATGAATG-CTGGGATGAAG BHQ2 & $\mathrm{rT}$ \\
\hline let7eto $\alpha$ & TGCGATCCTGAA-GTTTGACTCAAACTATACAACCTCCTACCTCA $p$ & cT \\
\hline let7cto $\alpha$ & TGCGATCCTGAA-GTTTGACTCAAACCATACAACCTACTACCTCA $p$ & cT \\
\hline mir92ato $\alpha$ & TGCGATCCTGAA-GTTTGACTCAAGCATTGCAACCGATCCCAACC $p$ & cT \\
\hline mir39toa & TGCGATCCTGAA-GTTTGACTCACAAGCTGATTTACACCC $p$ & cT \\
\hline let7ato $\beta$ & TGCAGTCCAGAA-GTTTGACTCAAACTATACAACCTACTACCTCA $p$ & cT \\
\hline let7eto $\beta$ & TGCAGTCCAGAA-GTTTGACTCAAACTATACAACCTCCTACCTCA $p$ & cT \\
\hline mir7to $\beta$ & TGCAGTCCAGAA-GTTTGACTCAAACAACAAAATCACTAGTCTTCCA $p$ & cT \\
\hline mir215to $\beta$ & TGCAGTCCAGAA-GTTTGACTCAGTCTGTCAATTCATAGGTCAT $p$ & cT \\
\hline mir203ato $\beta$ & TGCAGTCCAGAA-GTTTGACTCAACTAGTGGTCCTAAACATTTCAC $p$ & cT \\
\hline let7toy & TGCTGGGATGAA-GTTTGACTCAAACTATACAACCTACTACCTCA $p$ & cT \\
\hline$\alpha \mathrm{k} \beta$ & C*A*T*TCTGGACTGAAAA-CAATGACTCGATCCTGAA $p$ & kT \\
\hline$\alpha \mathrm{k} \beta A 0$ & C*A*T*TCTGGACTG-CAATGACTCGATCCTGAA $p$ & $\mathrm{kT}$ \\
\hline$\alpha \mathrm{k} \beta A 1$ & C*A*T*TCTGGACTGT-CAATGACTCGATCCTGAA $p$ & $\mathrm{kT}$ \\
\hline$\alpha \mathrm{k} \beta A 2$ & C*A*T*TCTGGACTGTT-CAATGACTCGATCCTGAA $p$ & kT \\
\hline$\alpha \mathrm{k} \beta A 3$ & C*A*T*TCTGGACTGTTT-CAATGACTCGATCCTGAA $p$ & $\mathrm{kT}$ \\
\hline$\alpha \mathrm{k} \beta A 4$ & C*A*T*TCTGGACTGTTTT-CAATGACTCGATCCTGAA $p$ & $\mathrm{kT}$ \\
\hline$\alpha k \beta A 5$ & C*A*T*TCTGGACTGTTTTT-CAATGACTCGATCCTGAA $p$ & kT \\
\hline$\beta k \alpha$ & C*A*T*TCAGGATCGAAAA-CAATGACTCAGTCCAGAA $p$ & kT \\
\hline$\alpha \mathrm{kp}$ & C*A*T*TCATCCCAGAAAA-CAATGACTCGATCCTGAA $p$ & $\mathrm{kT}$ \\
\hline$\gamma k \alpha$ & C*A*T*TCTGGACTGAAAA-CAATGACTCTGGGATGAA $p$ & kT \\
\hline$\beta k \gamma$ & C*A*T*TCATCCCAGAAAA-CAATGACTCAGTCCAGAA $p$ & kT \\
\hline$\gamma k \beta$ & C*A*T*TCAGGATCGAAAA-CAATGACTCTGGGATGAA $p$ & kT \\
\hline let7a & ugagguaguagguuguauaguu & microRNA \\
\hline let7e & ugagguaggagguuguauaguu & microRNA \\
\hline let7c & ugagguaguagguuguaugguu & microRNA \\
\hline mir39 & ucaccggguguaaaucagcuug & microRNA \\
\hline mir92a & agguugggaucgguugcaaugcu & microRNA \\
\hline mir7 & uggaagacuagugauuuuguuguu & microRNA \\
\hline mir215 & augaccuaugaauugacagac & microRNA \\
\hline mir203a & gugaaauguuuaggaccacuag & microRNA \\
\hline
\end{tabular}

